\section{Brain activity in chess playing}

SIR - There is widespread interest in identifying the topography of the neural networks subserving problem solving in humans. One potentially fruitful approach is to use functional neuroimaging techniques in conjunction with cognitive tasks ${ }^{1}$. Chess is an example of such $\mathrm{a} \operatorname{task}^{2}$, and we have administered chess problems in conjunction with $\left[{ }^{15} \mathrm{O}\right]$ waterpositron emission tomography (PET) to identify the neural network(s) activated in this process ${ }^{3}$.

The stimuli for all the task conditions were black-andwhite chess diagrams presented on a computer screen. To isolate brain activation changes related to the use of specific cognitive processes, we devised four conditions arranged in a subtractive hierarchy: (1) black/white discrimination, in which subjects had to indicate whether or not there were chessmen of a given colour on the board; (2) spatial discrimination, in which an $X$ was displayed in one of the board's squares and subjects had to identify the colour of the chessman closest to the $X$; (3) rule retrieval, in which subjects analysed a simple, single move, in responding to questions like "can the white knight capture a black rook?"; and (4) checkmate judgement, in which the subjects had to determine whether the player with a given colour could checkmate in one move. All answers were indicated by pressing response keys labelled yes or no. Ten righthanded males who had played chess for more than 4 years and were regularly participating in chess tournaments volunteered for this study.

When the results of the black/white discrimination condition were subtracted from the spatial discrimination condition, brain activation was observed bilaterally (see figure for a visual rendering of all the analyses) at the parieto-occipital lobe junction (areas 7 and 19), at the left middle temporal gyrus, and at the left superior premotor cortex. Extrastriate visual cortical areas are mainly organized around two anatomically separate and functionally specialized processing streams: a ventral occipito-temporal pathway for identifying objects; and a dorsal occipito-parietal pathway for perceiving the spatial relations between objects ${ }^{4.5}$. The black/white discrimination task only

required the subject to process figureground separation and discriminate between black and white chess pieces. The extra visual analysis required by the spatial discrimination condition explains the dorsal pathway activity. Activity in area 7 (both in the superior parietal lobule and in the medial superior parietal cortex) has

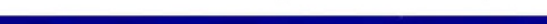

been repeatedly associated with spatial vision and with shifting spatial attention. We also found activation in a region of the left superior premotor cortex (superior area 6) which previous studies suggested was related to preparing a response to a selected peripheral location.

Subtraction of the spatial discrimination condition from the rule retrieval condition yielded several foci located

1. Roland. P. E. \& Friberg, L. J. Neurophysiol. 53, 1219 1243 (1985).

2. Charness, $\mathbf{N}$. in Towards a General Theory of Expertise: Prospects and Limits (eds Ericsson. K. A. \& Smith, J.) Prospects and Limits (eds Ericsson, K. A. \& Sm

3. Raichle, M. E. in Handbook of Physiology, Section 1: The Nervous System 5, 643-674 (1987).

4. Desimone, R. \& Ungerleider, L. G. in Handbook of Neuropsychology (eds Boller, F. \& Grafman, J.) 267-299 (Elsevier, Amsterdam, 1990)

5. Haxby, J. V. et al. Proc. natn. Acad. Sci. U.S.A. 88 , 1621-1625 (1991).

6. Mitner, B. Br. med. Bull. 27, 272-277 (1971)

7. Fiez, J. A., Petersen, S. E., Cheney, M. K. \& Raichle, M. E. Brain 115, 155-178 (1992)

8. Grafman, J. in Integrating Theory and Practice in Clinical Neuropsychology (ed. Perecman, E.) 93-138 (Eribaum Hillsdale, New Jersey, 1989)

9. Friston, K. J., Frith, C. D., Liddle, P. F. \& Frackoviak R.S.I.J. Cereb. Blood Fiow Metab. 11. 690-699 (1991)

10. Talairach, J. \& Tornoux, P. Co-planar Stereotaxic Atlas of the Human Brain (Thieme, Stuttgart, 1988)
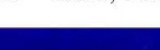
medial aspects of the left temporal lobe, the latter being mainly due to activation of the left hippocampus. We suggest that the retrieval of move sequences paired with a particular chess piece was responsible for the hippocampus and temporal lobe activation $^{6}$. A small peak of activation was also found in the inferior part of the post-central gyrus as well as in the cerebellum, which has recently been implicated in cognitive processing ${ }^{7}$.

In the subtraction of rule retrieval from checkmate the differential activity was confined to two large areas, the junction between the occipital and the parietal lobe, and the frontal lobe. We attribute the bilateral focus of activation we recorded at the occipitoparietal junction (areas 7, 18 and 19) to the act of generating sequential board images and to attentional shifts across the images. Activation recorded in the frontal eye fields (area 8) might be associated with an increase in extraocular movements during the instantiation of various endgame strategies. We also found increased activity in two prefrontal regions, one located in the left orbitofrontal cortex, and the second in the right prefrontal cortex. We believe that these two regions of activation subserve managerial knowledge required

ing and sequential execution for the planning and sec
of endgame strategies

We have demonstrated that solving a complex problem calls for the activity of a network of several interrelated, but functionally distinct, cerebral areas. The use of neuroimaging techniques such as PET, carefully coupled with distinctive information-processing demands, can help disentangle the role of each of these areas in human problem-solving activity.

\section{Paolo Nichelli*}

Jordan Grafman ${ }^{\dagger}$

Pietro Pietrini ${ }^{\ddagger}$

David Alway

John C. Carton

Robert Miletich

Cognitive Neuroscience Section,

Medical Neurology Branch,

National Institute of Neurological

Disorders and Stroke,

Bldg 10; Room 5\$209,

National Institute of Aging ${ }^{\ddagger}$

National Institutes of Health,

Bethesda, Maryland 20892, USA

*Also at: Clinica Neurologica, Univ. Modena, Italy. ${ }^{\dagger}$ Author for correspondence. 